\title{
On uniqueness of JSJ decompositions of finitely generated groups
}

Max Forester*

\begin{abstract}
We give an example of two JSJ decompositions of a group that are not related by conjugation, conjugation of edge-inclusions, and slide moves. This answers the question of Rips and Sela stated in [RS].

On the other hand we observe that any two JSJ decompositions of a group are related by an elementary deformation, and that strongly slide-free JSJ decompositions are genuinely unique. These results hold for the decompositions of Rips and Sela, Dunwoody and Sageev, and Fujiwara and Papasoglu, and also for accessible decompositions.
\end{abstract}

Mathematics Subject Classification (2000). 20F65, 20E08, 57M07.

Keywords. G-tree, JSJ decomposition, splitting, Baumslag-Solitar group, accessible group.

\section{Introduction}

In this paper we discuss the extent to which JSJ decompositions of groups are unique. In [Sel] and [RS] it was shown that if $G$ is a (Gromov) hyperbolic group then any two JSJ decompositions of $G$ must be related by conjugation, conjugation of edge-inclusions, and slide moves. Rips and Sela also noted that the same uniqueness statement holds in many other cases. However, the general case was left open as a question. Here we give examples of JSJ decompositions of a finitely presented group that are not related by such moves, answering their question in the negative.

In light of the examples it is natural to ask what form of uniqueness does hold for finitely presented groups. It turns out that JSJ decompositions are unique up to elementary deformation, a notion that is studied extensively in $[\mathrm{F}]$. Furthermore, if a decomposition is strongly slide-free then it is genuinely unique. As we will see in Section 3, these results follow directly from the more general results in $[F]$. The same uniqueness results also hold for accessible (or one-ended) decompositions.

In this paper we focus mainly on the JSJ decomposition of Rips and Sela,

\footnotetext{
${ }^{*}$ Research supported by EPSRC grant GR/N20867.
} 
though our results apply equally well to the JSJ decompositions of Dunwoody and Sageev [DS] and Fujiwara and Papasoglu [FP]. It seems worthwhile to mention the uniqueness properties of other related decompositions.

There have been several constructions, originally inspired by the canonical decompositions of 3-manifolds due to Jaco and Shalen, and Johannson [JaS, Jo]. The first result of this kind was Kropholler's decomposition for Poincaré duality groups in $[\mathrm{K}]$. This decomposition is unique. Sela defined a JSJ decomposition for torsion free hyperbolic groups in [Sel], and Bowditch found an equivalent topological construction for one-ended hyperbolic groups; this decomposition is essentially unique [Bo, Si]. Next came the three decompositions already mentioned [RS, DS, FP], whose uniqueness properties are discussed in this paper. Finally there is a recent construction due to Scott and Swarup, described in the 3-manifold case in [ScS1], and in the general case in [ScS2]. This decomposition is unique and it agrees with the topological JSJ decomposition in the case of a 3-manifold group. On the other hand, the JSJ decompositions considered here are sometimes finer, and can reveal more information about the group.

The paper is organized as follows. In Section 1 we discuss moves between decompositions. In Section 2 we present the main examples, which are generalized Baumslag-Solitar trees. Most of the section is devoted to showing that such trees qualify as JSJ decompositions, under mild assumptions (Theorem 2.15 and Proposition 2.17). This result is interesting in its own right. In Section 3 we discuss our uniqueness result, Theorem 3.1.

Acknowledgements. I would like to thank Gilbert Levitt for pointing out that the results of $[F]$ could be applied to JSJ decompositions. Similar suggestions were made by M. Sageev, Z. Sela, and G. A. Swarup. During this work I benefitted from discussions and correspondence with Peter Scott and G. A. Swarup. I also thank David Epstein for his encouragement, and the referee for suggesting improvements to the exposition.

\section{Preliminaries}

We will use Serre's notation for graphs and trees. Thus a graph $A$ is a pair of sets $(V(A), E(A))$ with maps $\partial_{0}, \partial_{1}: E(A) \rightarrow V(A)$ and $e \mapsto \bar{e}$ (for $e \in E(A)$ ), such that $\partial_{i} \bar{e}=\partial_{1-i} e$ and $e \neq \bar{e}$ for all $e$. An element $e \in E(A)$ is to be thought of as an oriented edge with initial vertex $\partial_{0} e$ and terminal vertex $\partial_{1} e$.

Let $G$ be a group. A $G$-tree is a tree with a $G$-action by automorphisms, without inversions. There is a correspondence between $G$-trees and graphs of groups having fundamental group $G$, as explained in [Ser]. We will consider certain moves between graphs of groups that do not change the fundamental group. Equivalently, these are moves between $G$-trees. A more complete discussion of these moves is given in $[\mathrm{F}, \S 3]$. 
Definition 1.1. In a collapse move, an edge in a graph of groups carrying an amalgamation of the form $A *_{C} C$ is collapsed to a vertex with group $A$. Every inclusion map having target group $C$ is reinterpreted as a map into $A$, via the injective map of vertex groups $C \hookrightarrow A$. This move simplifies the underlying graph without enlarging any vertex or edge groups.

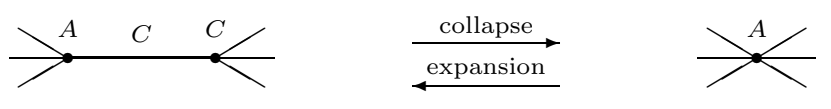

An expansion move is the reverse of a collapse move. Both of these moves are called elementary moves. An elementary deformation is a finite sequence of such moves. A graph of groups is reduced if it admits no collapse moves. This means that if an inclusion map from an edge group to a vertex group is an isomorphism, then the edge is a loop.

Definition 1.2. The elementary deformation shown below, consisting of an expansion move followed by a collapse, is called a slide move. In order to perform the expansion it is required that $D \subseteq C$ (regarded as subgroups of $A$ ).

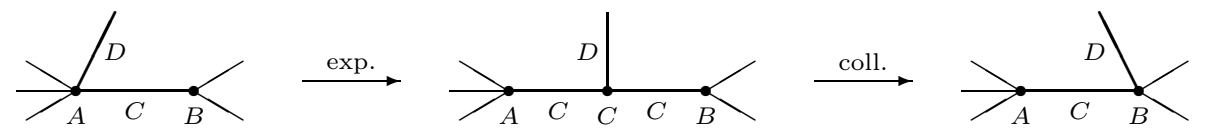

It is permitted for the edge carrying $C$ to be a loop; in this case the only change to the graph of groups is in the inclusion map $D \hookrightarrow A$. See Proposition 2.1 for an example.

Definition 1.3. A fold is most easily described in terms of $G$-trees. The graph of groups description involves many different cases which are explained in $[\mathrm{BF}]$. To perform a fold in a $G$-tree one chooses edges $e$ and $f$ with $\partial_{0} e=\partial_{0} f$, and identifies $e$ and $f$ to a single edge. One also identifies $\gamma e$ with $\gamma f$ for every $\gamma \in G$, so that the resulting quotient graph has a $G$-action. It is not difficult to show that the new graph is a tree.

Definition 1.4. A generalized Baumslag-Solitar tree is a $G$-tree whose vertex and edge stabilizers are all infinite cyclic. The groups $G$ that arise are called generalized Baumslag-Solitar groups. Examples include the classical Baumslag-Solitar groups and torus knot groups. When discussing specific examples it is convenient to use edge-indexed graphs, as seen in the next section. They depict graphs of groups in which all edge and vertex groups are $\mathbb{Z}$. The indices define the inclusion maps, which are simply multiplication by various non-zero integers. 


\section{Two JSJ decompositions not related by slide moves}

In [RS, p. 106], Rips and Sela ask whether any two JSJ decompositions of a group must be related by conjugation, conjugation of edge-inclusions, and slide moves. If one regards the JSJ decomposition as a $G$-tree then the first two modifications have no effect (up to $G$-isomorphism). Thus, they are asking whether two such $G$-trees are related by slide moves. We show by example that this need not be the case.

The examples are generalized Baumslag-Solitar trees. We will describe two such trees and show that they are related by an elementary deformation. This implies that the two groups are the same. Then we verify that the trees are not slide-equivalent, and that they represent JSJ decompositions of their common group.

Proposition 2.1. If an elementary move is performed on a generalized BaumslagSolitar tree, then the quotient graph of groups changes locally as follows:

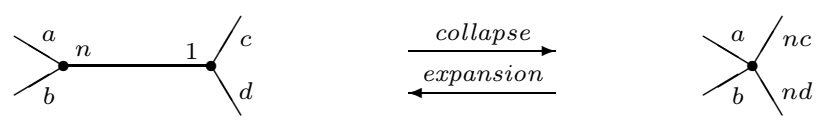

A slide move has the following description:
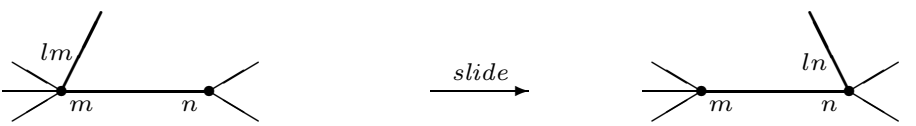

or
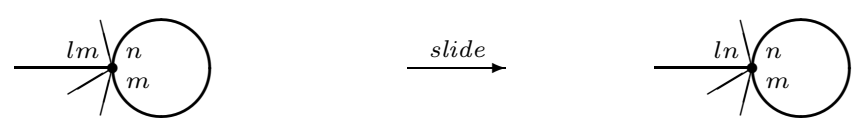

The proof is straightforward and is left to the reader.

Main Example. Choose non-zero integers $m, n, r$, and $s$. The following diagrams depict a sequence of elementary deformations between generalized BaumslagSolitar trees. The initial and final trees are the examples that interest us. Call them $X$ and $Y$ respectively, and let $G$ be the group.

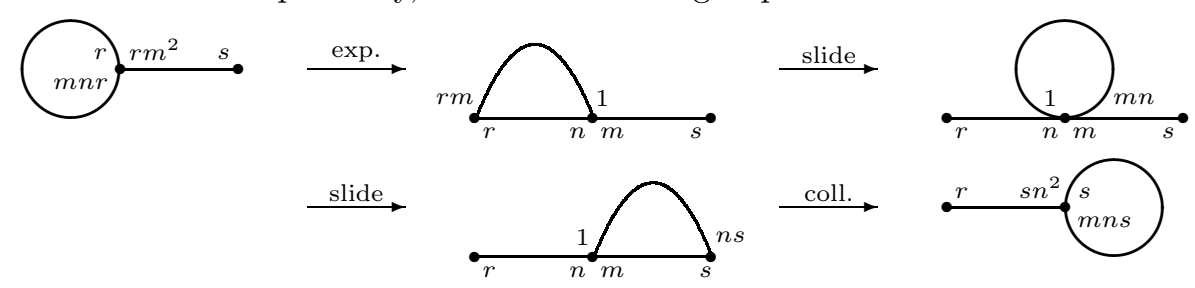


Proposition 2.2. If $m \nmid n$ and $n \nmid m$ then $X$ and $Y$ are not related by slide moves.

Proof. Note that $m \neq \pm 1$, and so $r m^{2} \nmid m n r, m n r \nmid r m^{2}$, and $r m^{2} \nmid r$. Thus $X$ admits only one slide move, in which the free edge travels around the loop. This move changes the index $\mathrm{rm}^{2}$ to $\mathrm{rm}^{3} n$. Repeating this move, the index becomes $r m^{k+2} n^{k}$ with $k \geqslant 0$. Call this $G$-tree $X_{k}$. Then since $r m^{k+2} n^{k} \nmid m n r$ and $r m^{k+2} n^{k} \nmid r$, the only slide moves available from $X_{k}$ are those resulting in $X_{k+1}$ and $X_{k-1}($ when $k \geqslant 1)$. Since $Y ¥ X_{k}$ for any $k$, the result follows.

Remark 2.3. If one considers the set of reduced $G$-trees for a given group $G$, then the example shows that the relation of slide-equivalence may be strictly finer than the relation of elementary deformation.

Throughout the rest of this section we verify that $X$ and $Y$ are in fact JSJ decompositions of $G$. This will be the case as long as $r, s \neq \pm 1$. First we review some basic properties of generalized Baumslag-Solitar groups. The key property, from the point of view of JSJ decompositions, is given in Lemma 2.9.

Definition 2.4. Let $X$ be a $G$-tree. An element $\gamma \in G$ is elliptic if it fixes a vertex of $X$ and hyperbolic otherwise. If $\gamma$ is hyperbolic then there is a unique $\gamma$-invariant line in $X$, called the axis of $\gamma$, on which $\gamma$ acts as a translation [Ser, Chapter I, Proposition 24]. From this description it is clear that for any $n \neq 0$, the element $\gamma^{n}$ is hyperbolic if and only if $\gamma$ is, and when this occurs they have the same axis.

Two elements $\gamma, \delta \in G$ are commensurable if there exist non-zero integers $m, n$ such that $\gamma^{m}=\delta^{n}$. Note that commensurable hyperbolic elements have the same axis. The commensurator of $\gamma$ is the set of all elements $\delta \in G$ such that $\delta \gamma \delta^{-1}$ and $\gamma$ are commensurable.

Lemma 2.5. Let $X$ be a $G$-tree. If $\gamma \in G$ is hyperbolic then its commensurator stabilizes its axis. If $\gamma \in G$ is elliptic and $X$ is a generalized Baumslag-Solitar tree, then the commensurator of $\gamma$ is all of $G$.

Proof. Suppose that $\delta \gamma \delta^{-1}$ and $\gamma$ are commensurable, where $\gamma$ is hyperbolic with axis $L$. Then $\delta \gamma \delta^{-1}$ also has axis $L$. However, the axis of $\delta \gamma \delta^{-1}$ is $\delta L$, and hence $\delta$ stabilizes $L$. Next suppose that $X$ is a generalized Baumslag-Solitar tree. Then all non-trivial elliptic elements are commensurable, and hence every elliptic element is commensurable with all of its conjugates.

Lemma 2.6. Let $X$ be a generalized Baumslag-Solitar tree with group $G ¥ \mathbb{Z}$. Then:

(a) $G$ is not free;

(b) $G$ is torsion-free and has cohomological dimension 2;

(c) $G$ has one end, if it is finitely generated; 
(d) $X$ contains a $G$-invariant line if and only if $G$ is isomorphic to $\mathbb{Z} \times \mathbb{Z}$ or the Klein bottle group.

Proof. For (a), first suppose that any two vertex stabilizers are contained in an infinite cyclic subgroup. If there is a maximal stabilizer $C$, then $G \cong C \times \pi_{1}(G \backslash X)$, which is either $\mathbb{Z}$ or non-free. If there is no maximal stabilizer, then the set of elliptic elements is an abelian, non-finitely generated subgroup of $G$. Free groups contain no such subgroups. Finally, if there are two stabilizers not contained in an infinite cyclic subgroup, let $\gamma$ and $\delta$ be their generators, respectively. Then $\gamma$ and $\delta$ cannot generate a free subgroup of $G$ because they are commensurable.

Claim (b) follows from the fact that $G$ is the fundamental group of a space $Z$ with universal cover homeomorphic to $X \times \mathbb{R}$. The space $Z$ is the total space of a graph of spaces in which every vertex and edge space is a circle (see [ScW] for these notions). Note that $Z$ is aspherical and 2-dimensional.

For (c), if $G$ is finitely generated then it acts cocompactly on the minimal subtree of $X$. Replace $X$ by this subtree. Then the space $Z$ is compact, and so $G$ and $X \times \mathbb{R}$ have the same number of ends. The space $X \times \mathbb{R}$ has one end unless $X$ is compact, but this does not occur because $G \neq \mathbb{Z}$.

For (d), if $X$ has an invariant line then $G$ acts on this line with infinite cyclic stabilizers. Since $G \neq \mathbb{Z}$, this action is cocompact. Hence $G=\mathbb{Z} * \mathbb{Z}$ or $G=\mathbb{Z} * \mathbb{Z}$, with inclusion maps multiplication by \pm 2 in the first case and \pm 1 in the second. For the converse, if $G \cong \mathbb{Z} \times \mathbb{Z}$ or the Klein bottle group, then no minimal subtree can have more than two ends, for this would imply that $G$ has exponential growth.

Lemma 2.7. Let $X$ be a generalized Baumslag-Solitar tree with group G. Every subgroup $H$ of $G$ is either a generalized Baumslag-Solitar group or a free group (and not both except for $\mathbb{Z}$ ). If $H$ is free and non-abelian then every non-trivial element of $H$ is hyperbolic.

Proof. Every edge stabilizer has finite index in its neighboring vertex stabilizers, and hence $X$ is locally finite. This implies that for every group action on $X$, all vertex and edge stabilizers are commensurable. Thus the stabilizers of $H$ acting on $X$ are either all infinite cyclic or all trivial. If the former occurs then $H$ is not free unless it is $\mathbb{Z}$, by Lemma 2.6(a). Hence if $H$ is free and non-abelian then its stabilizers in $X$ are all trivial.

Definition 2.8. A splitting of $G$ over $C$ is a graph of groups decomposition of the form $G=A *_{C} B$ with $A \neq C \neq B$, or $G=A *_{C}$ (with no restriction on $C$ ). We also say that $G$ splits over $C$. If $\mathrm{A}_{1}$ is a splitting of $G$ over $C$ and $\mathrm{A}_{2}$ is another graph of groups decomposition of $G$, we say that $\mathrm{A}_{1}$ is hyperbolic in $\mathrm{A}_{2}$ if some element of $C$ is hyperbolic in the action of $G$ on the Bass-Serre tree of $\mathrm{A}_{2}$.

Lemma 2.9. Let $X$ be a generalized Baumslag-Solitar tree with group G. Assume 
that $X$ contains no $G$-invariant point or line. If $G$ splits over a 2 -ended group $C$, then $C$ is contained in a vertex stabilizer of $X$.

Proof. Let $T$ be the Bass-Serre tree for the splitting over $C$, and let $e$ be an edge of $T$ with stabilizer $C$. Note that $T$ contains no invariant point or line, for this would imply that $G \cong \mathbb{Z}$, or that $G$ splits as $A_{1} *_{C} B_{1}$ with $\left[A_{1}: C\right]=\left[B_{1}: C\right]=2$, or $A_{1} *_{C}$ with both inclusions surjective. In these cases $G=\mathbb{Z} \times \mathbb{Z}$ or the Klein bottle group, contradicting the hypotheses (by Lemma 2.6(d)).

Let $\gamma \in G$ be a generator of a vertex stabilizer in $X$. By Lemma 2.5 the commensurator of $\gamma$ is all of $G$. Now consider the action of $G$ on $T$. If $\gamma$ is hyperbolic then its commensurator stabilizes its axis, but we have observed that $G$ does not stabilize any line in $T$. Hence $\gamma$ fixes a vertex $v$ of $T$. Now let $\delta \in G$ be chosen so that $e$ separates $v$ from $\delta v$. Since $\gamma$ and $\delta \gamma \delta^{-1}$ are commensurable, some power of $\gamma$ fixes both $v$ and $\delta v$, and also $e$. Thus $\gamma^{n} \in C$ for some $n$. Note that $C$ is infinite cyclic because it is torsion free and 2-ended. Letting $c$ be a generator, we have that $c^{m}=\gamma^{n}$ for some $m$. Since this element is elliptic in $X, c$ is also elliptic in $X$.

Definition 2.10. In order to discuss JSJ decompositions we need a further definition, from [RS]. A subgroup $H$ of $G$ is quadratically hanging if $H$ is isomorphic to the fundamental group of a compact 2-orbifold with boundary, and there exists a minimal $G$-tree with the following properties: all edge stabilizers are infinite cyclic, there is a vertex $v$ with stabilizer $H$, and the stabilizers of the edges incident to $v$ are precisely the conjugates (in $H$ ) of the peripheral subgroups of $H$. It is also required that the 2 -orbifold have negative Euler characteristic and contain a pair of intersecting "weakly essential" simple closed curves.

Lemma 2.11. Let $G$ be a generalized Baumslag-Solitar group. Then $G$ contains no quadratically hanging subgroups.

Proof. Let $X$ be a generalized Baumslag-Solitar tree with group $G$. Suppose that $H \subseteq G$ is a quadratically hanging subgroup with corresponding $G$-tree $T$. Since $G$ is torsion-free, $H$ is the fundamental group of a compact surface with boundary and negative Euler characteristic. It is conceivable that the surface is closed, in which case $T$ is a point and $H=G$. However, no generalized Baumslag-Solitar group is isomorphic to a closed surface group of this type. For example, no generalized Baumslag-Solitar group is hyperbolic, except for $\mathbb{Z}$.

Thus, we can assume that the surface has non-empty boundary, so that $H$ is a non-abelian free group. In particular $G$ is not isomorphic to $\mathbb{Z}, \mathbb{Z} \times \mathbb{Z}$, or the Klein bottle group, and so $X$ contains no $G$-invariant point or line. Now let $C \subseteq H$ be a peripheral subgroup, and consider the splitting of $G$ over $C$ arising from the $G$-tree $T$. By Lemma 2.9, every element of $C$ is elliptic relative to $X$. This contradicts Lemma 2.7. 
Definition 2.12. The next theorem [RS, Theorem 7.1] defines the notion of a JSJ decomposition. To be specific, a JSJ decomposition of a group is a graph of groups decomposition satisfying the properties of $\mathrm{G}$ in Theorem 2.13. The precise definition of $C M Q$ subgroup is not important here, except that it is a quadratically hanging subgroup with additional properties. Similarly, we omit the definition of weakly essential simple closed curve.

A splitting $\mathrm{A}_{1}$ of $G$ is unfolded if there do not exist a splitting $\mathrm{A}_{2}$ and a nontrivial fold $\mathrm{A}_{2} \rightarrow \mathrm{A}_{1}$. Note that such a fold involves only $G$-trees having a single edge orbit, and therefore induces an isomorphism of quotient graphs. A graph of groups $G_{1}$ is unfolded if every splitting arising from its edges is unfolded in the above sense. Note that this is very different from requiring no non-trivial fold $\mathrm{G}_{2} \rightarrow \mathrm{G}_{1}$ to exist.

Theorem 2.13 (Rips-Sela). Let $G$ be a finitely presented group with one end. There exists a reduced, unfolded graph of groups decomposition $\mathrm{G}$ of $\mathrm{G}$ with infinite cyclic edge groups, such that the following conditions hold.

(a) Every canonical maximal quadratically hanging (CMQ) subgroup of $G$ is conjugate to a vertex group of $\mathrm{G}$. Every quadratically hanging subgroup of $G$ can be conjugated into one of the $C M Q$ subgroups of $G$. Every non-CMQ vertex group of $\mathrm{G}$ is elliptic in every graph of groups decomposition of $G$ having infinite cyclic edge groups.

(b) A splitting of $G$ over $\mathbb{Z}$ which is hyperbolic in another splitting over $\mathbb{Z}$ is obtained from $\mathrm{G}$ by cutting a 2-orbifold corresponding to a CMQ subgroup of $G$ along a weakly essential simple closed curve.

(c) Let A be a splitting of $G$ over $\mathbb{Z}$ which is elliptic with respect to every other splitting over $\mathbb{Z}$. Then there exists a $G$-equivariant simplicial map from a subdivision of $T_{\mathrm{G}}$, the Bass-Serre tree of $\mathrm{G}$, to $T_{\mathrm{A}}$ (the Bass-Serre tree of $\mathrm{A})$.

(d) Let A be a graph of groups decomposition of $G$ with infinite cyclic edge groups. Then there exist a decomposition $\mathrm{G}^{\prime}$ obtained from $\mathrm{G}$ by splitting the CMQ subgroups along weakly essential simple closed curves on their corresponding 2-orbifolds, and a G-equivariant simplicial map from a subdivision of $T_{\mathrm{G}^{\prime}}$ to $T_{\mathrm{A}}$.

Rips and Sela also include a uniqueness statement in their theorem, which we discuss in Section 3.

Remark 2.14. Our notion of "reduced" $G$-trees is stronger than the definition used by Rips and Sela, and stated in $[\mathrm{BF}]$. However it is true that if one performs collapse moves on a JSJ decomposition then the result is again a JSJ decomposition. Thus Theorem 2.13 is valid with our definition. This issue will be relevant when we discuss uniqueness in the next section. 
Theorem 2.15. Let $X$ be a generalized Baumslag-Solitar tree with group G. If $X$ is reduced, unfolded, and is not a point or line, then $X$ is a JSJ decomposition of $G$.

Proof. Reduced trees are minimal, so $X$ contains no invariant point or line. The first two statements of (a) are vacuously true, by Lemma 2.11. The third statement is a consequence of Lemma 2.9, as follows. Let A be a graph of groups decomposition of $G$ with infinite cyclic edge groups. Choose an edge group $C \subseteq G$ of A. Then $C$ is elliptic in $X$ by Lemma 2.9. As $C$ is infinite cyclic, each vertex stabilizer of $X$ is commensurable with $C$. Therefore each vertex stabilizer of $X$ is elliptic in A. Claim (b) is also vacuously true by Lemma 2.9; if $G$ splits over infinite cyclic subgroups $C_{1}$ and $C_{2}$, then the lemma implies that $C_{1}$ and $C_{2}$ are commensurable, and hence neither splitting is hyperbolic in the other. Claim (c) is a special case of (d), since there are no CMQ subgroups. To prove (d) it suffices to verify that every vertex stabilizer of $X$ is elliptic in A. This was just shown in the proof of (a).

Remark 2.16. A similar argument shows that any generalized Baumslag-Solitar tree as above is also a JSJ decomposition over 2-ended groups in the sense of Dunwoody and Sageev [DS], and a JSJ decomposition over slender groups in the sense of Fujiwara and Papasoglu [FP].

Proposition 2.17. Let $X$ be a cocompact generalized Baumslag-Solitar tree with group $G$. If every edge stabilizer is a proper subgroup of its neighboring vertex stabilizers then $X$ is unfolded.

The assumption of cocompactness is not necessary, but it allows for a simpler proof.

Proof. Let $e$ be an edge of $X$ and let $\widehat{X}$ be the tree obtained from $X$ by collapsing each connected component of $X-G e$ to a vertex. Call this quotient map $q: X \rightarrow$ $\widehat{X}$. Then $\widehat{X}$ is the Bass-Serre tree corresponding to the splitting of $G$ associated to $e$. Now suppose that there is a non-trivial fold $f: Y \rightarrow \widehat{X}$. Let $e^{\prime} \in E(Y)$ be an edge with $f\left(e^{\prime}\right)=q(e)$. Without loss of generality, assume that the fold occurs at $\partial_{0} e^{\prime}$.

Consider the stabilizer of the vertex $\partial_{1} e$ in $X$, and let $\gamma$ be a generator. Then $G_{e}$ is generated by $\gamma^{n}$ for some $n$. Also $G_{e^{\prime}} \subsetneq G_{q(e)}=G_{e}$ and $G_{e^{\prime}} \neq\{1\}$, as $G$ has one end (by cocompactness and Lemma 2.6(c)). Thus $G_{e^{\prime}}$ is generated by $\gamma^{m n}$ for some $m>1$. This implies that $\gamma^{n} e^{\prime} \neq e^{\prime}$, and $\partial_{0} \gamma^{n} e^{\prime}=\partial_{0} e^{\prime}$ because the fold occurs at $\partial_{0} e^{\prime}$. The edges $\gamma^{n} e^{\prime}$ and $e^{\prime}$ separate $Y$ into three connected components, adjacent to the vertices $\partial_{0} e^{\prime}, \partial_{1} e^{\prime}$, and $\partial_{1} \gamma^{n} e^{\prime}$. Call these subtrees $Y_{0}$, $Y_{1}$ and $\gamma^{n}\left(Y_{1}\right)$ accordingly. It is clear that $\gamma$ fixes no point of $Y_{1}$ or $\gamma^{n}\left(Y_{1}\right)$. Also define the subtrees $\widehat{X}_{0}, \widehat{X}_{1}, X_{0}$, and $X_{1}$ similarly, as the connected components of 
$\widehat{X}-q(e)$ and $X-e$.

We claim that $\gamma e=e$, and hence $G_{e}=G_{\partial_{1} e}$. Note that since $\gamma^{m n}$ is elliptic in $Y$, so is $\gamma$. Therefore $\gamma$ fixes a vertex in $Y_{0}$. By equivariance of $f$ it also fixes a vertex $v$ of $\widehat{X}_{0}$. Then $\gamma$ stabilizes the subtree $q^{-1}(v) \subseteq X_{0}$. Since $e$ separates $q^{-1}(v)$ from $\partial_{1} e$ and $\gamma$ stabilizes both, $\gamma$ also fixes $e$.

Finally we return to the main example. If $r, s \neq \pm 1$ then $X$ and $Y$ are reduced and unfolded. Since neither tree is a point or line, both are JSJ decompositions of $G$.

\section{Uniqueness of decompositions}

We have seen that JSJ decompositions of a given group need not be related by slide moves. However, the results of $[\mathrm{F}]$ imply that they are unique up to elementary deformation, and also that many JSJ decompositions are genuinely unique. The reasoning applies to other decompositions as well: the JSJ decompositions of Dunwoody and Sageev [DS] and Fujiwara and Papasoglu [FP], and also accessible (or one-ended) decompositions.

The other JSJ decompositions just mentioned are defined in a similar way to the Rips-Sela version, by means of a kind of universal property. In Theorem 2.13 this property refers to splittings over infinite cyclic groups. The JSJ decomposition has edge groups of this type, and it provides a simultaneous description of every such splitting. In a similar fashion, the JSJ decomposition of Fujiwara and Papasoglu deals with splittings over slender groups. (A group is slender if every subgroup is finitely generated.) For the Dunwoody-Sageev decomposition one must first choose a "closed class" C of slender groups, and then the JSJ decomposition refers to splittings over elements of C. For example, C could be the class of 2-ended groups, or the class of finite extensions of $\mathbb{Z} \times \mathbb{Z}$. For further details on these JSJ decompositions see [DS] and [FP].

As mentioned earlier we require JSJ decompositions to be reduced, in order to apply Theorem 3.3 below. In all three versions this is easily arranged by performing collapse moves, though in the case of the Dunwoody-Sageev decomposition a small modification is required. Namely, we must use their decomposition $G_{\text {red }}$ rather than $\mathrm{G}$, which is obtained from $\mathrm{G}_{\text {red }}$ by subdivision.

An accessible decomposition is a reduced graph of groups whose edge groups are finite and whose vertex groups each have at most one end. Dunwoody showed in $[D]$ that every finitely presented group has an accessible decomposition.

The property shared by all of these decompositions is that any two particular decompositions (of the same kind) have the same elliptic subgroups. Here, an elliptic subgroup is any subgroup that fixes a vertex of the given $G$-tree. To state our uniqueness theorem we need one additional notion. A $G$-tree is strongly slidefree if it is minimal and, for all edges $e$ and $f$ with $\partial_{0} e=\partial_{0} f, G_{e} \subseteq G_{f}$ implies 
$f \in G e$. In terms of graphs of groups this means that for every vertex group $A$, if $C$ and $C^{\prime}$ are neighboring edge groups then no conjugate (in $A$ ) of $C$ is contained in $C^{\prime}$. Many graphs of groups arising in nature have this property, though there are obvious exceptions such as ascending HNN extensions.

Theorem 3.1. Let $G$ be a finitely generated group. Suppose that $X$ and $Y$ are $G$-trees representing decompositions of one of the following types: JSJ decompositions in the sense of Rips and Sela, Dunwoody and Sageev, or Fujiwara and Papasoglu, or accessible decompositions. Then $X$ and $Y$ are related by an elementary deformation. If $X$ is strongly slide-free then there is a unique $G$-isomorphism $X \rightarrow Y$.

Thus, in each case, strongly slide-free decompositions are genuinely unique. The proof is a direct application of the following two results [F, Theorems 1.1 and $1.2]$.

Theorem 3.2. Let $G$ be a group and let $X$ and $Y$ be cocompact $G$-trees. Then $X$ and $Y$ are related by an elementary deformation if and only if they have the same elliptic subgroups.

Theorem 3.3. Let $X$ and $Y$ be cocompact $G$-trees that are related by an elementary deformation. If $X$ is strongly slide-free and $Y$ is reduced then there is a unique $G$-isomorphism $X \rightarrow Y$.

Proof of Theorem 3.1. Both trees are cocompact because $G$ is finitely generated. Now it suffices to verify that the elliptic subgroups for $X$ and $Y$ agree. An equivalent property is that there exist equivariant maps between $X$ and $Y$ in each direction. This is proved in [RS, Theorem 7.1(v)] and [DS, p. 43] respectively for the first two types of decomposition. The third case is similar to these, and it can be derived formally from the main theorem of [FP].

For accessible decompositions we argue as follows. Let $G_{x}$ be a vertex stabilizer in $X$ and consider its action on $Y$. This action has finite edge stabilizers, and since $G_{x}$ has at most one end, the action must be trivial. Thus, every elliptic subgroup in $X$ fixes a vertex in $Y$, and conversely by symmetry.

\section{References}

[BF] M. Bestvina and M. Feighn, Bounding the complexity of simplicial group actions on trees, Invent. Math. 103 (1991), 449-469.

[Bo] B. H. Bowditch, Cut points and canonical splittings of hyperbolic groups, Acta Math. 180 (1998), no. 2, 145-186.

[D] M. J. Dunwoody, The accessibility of finitely presented groups, Invent. Math. 81 (1985), 449-457. 
[DS] M. J. Dunwoody and M. E. Sageev, JSJ-splittings for finitely presented groups over slender groups, Invent. Math. 135 (1999), 25-44.

[F] M. Forester, Deformation and rigidity of simplicial group actions on trees, Geom. Topol. 6 (2002), 219-267.

[FP] K. Fujiwara and P. Papasoglu, JSJ-decompositions of finitely presented groups and complexes of groups, preprint, July 1998.

[JaS] W. Jaco and P. B. Shalen, Seifert fibered spaces in 3-manifolds, Memoirs of the Amer. Math. Soc. 220 (1979)

[Jo] K. Johannson, Homotopy equivalences of 3-manifolds with boundaries, Lecture Notes in Mathematics, vol. 761, Springer-Verlag, 1979.

[K] P. H. Kropholler, An analogue of the torus decomposition theorem for certain Poincaré duality groups, Proc. London Math. Soc. (3) 60 (1990), 503-529.

[RS] E. Rips and Z. Sela, Cyclic splittings of finitely presented groups and the canonical JSJ decomposition, Ann. of Math. 146 (1997), 53-109.

[ScS1] P. Scott and G. A. Swarup, Canonical splittings of groups and 3-manifolds, Trans. Amer. Math. Soc. 353 (2001), 4973-5001.

[ScS2] P. Scott and G. A. Swarup, Regular neighbourhoods and canonical decompositions for groups, preprint, arXiv:math.GR/0110210.

[ScW] P. Scott and T. Wall, Topological methods in group theory, Homological Group Theory (C. T. C. Wall, ed.), LMS Lecture Note Series, vol. 36, Cambridge University Press, 1979, 137-203.

[Sel] Z. Sela, Structure and rigidity in (Gromov) hyperbolic groups and discrete groups in rank 1 Lie groups II, Geom. Funct. Anal. 7 (1997), 561-593.

[Ser] J.-P. Serre, Trees, Springer-Verlag, 1980.

[Si] S. Singh, Uniqueness of the JSJ decomposition for negatively curved groups, Ph.D. thesis, The University of Michigan, 1999.

Max Forester

Mathematics Institute

University of Warwick

Coventry, CV4 7AL

UK

e-mail: forester@maths.warwick.ac.uk

(Received: October 19, 2001) 